\title{
Research on Method of Random Error Separation Based on Wavelet Transform and Frequency bands Character
}

\author{
Xiuyun DUAN \\ PLA 91550 \\ Unit 94 \\ Dalian, China \\ macolobo@gmail.com
}

\author{
Yu HUANG \\ PLA 91550 \\ Unit 94 \\ Dalian, China \\ huangyu1025@sina.com
}

\begin{abstract}
- a new method of random error separation based on wavelet transform and the frequency bands character of measurement data is proposed. Approximates the random error with the detail components of the wavelet decomposition, determines the optimal decomposition level on the frequency intervals between the random errors and other components, and finally gets the random error by reconstructing all the detail components. The method is simply and efficient, avoids the indirect error with modeling and indirect methods, and computer simulation proves the robustness of the separation results.
\end{abstract}

Keywords-component; wavelet transform; frequency bands character; random error separation

\section{INTRODUCTION}

Random errors and fixed errors are the main errors in the measuring data. It is a very important job to separate the random errors, which is helpful to estimate the true value and analysis the fixed errors. Affected by the measuring equipments and conditions, the measuring data sometimes exhibit bad SNR (Signal Noise Ratio) with large error value which often don't accord with white noise model, and the routine methods as follows can't get satisfying separating results.

The variate-difference method and the least square fitting method are classic methods to calculate the random error, which discussed in [1]. However, these two methods are not competent for the data with large and complicated errors. The autoregressive-moving average model statics method, applied in [2] is applicable for the data with explicit error models, which can't be practical for the most instances. Other regressive statics methods see in [3] need the user with professional knowledge of mathematics and modeling.

As a powerful tool of signal analysis, wavelet transform has good localization properties in time and frequency domain, and can focus to any details of the analysis object with taking fine time or frequency step length of high frequency, express any changes existing in the object, so as to get accurate random error separation results from the measurement data with bad SNR, see in [4] [9].

By using wavelet transform to separate the random error, the key process lies in the determination of optimal decomposition levels. On the one hand, we want to separate the random error components as far as possible, on the other hand, keep the fixed errors and true value apart from the separated random errors. The current methods need either manual setting threshold control or results testing with extracted trend by wavelet transform, which increase the difficulty of the application of separation methods and raise the risk of error introduced. In accordance with the above case, the paper proposes a new method which approximates the random errors with the detail components of the wavelet decomposition, determines the optimal decomposition level on the frequency intervals between the random errors and other components, then gets the random error directly. The method avoids the indirect error with modeling and indirect methods, and computer simulation proves its simplicity and efficiency.

\section{WAVELET TRANSFORM OF THE MEASUREMENT DATA}

\section{A. Summarize of Wavelet Transform}

Wavelet transform is made up of wavelet decomposition and wavelet reconstruction. The decomposition process can be iterated, with successive approximations being decomposed in turn, so that one signal is broken down into many lower resolution components. This is called the wavelet decomposition tree.

Fig. 1 shows the tree diagram of signal $S$ after three levels wavelet decomposition. $S$ denotes the signal to be decomposed, $A i$ denotes approximation components of decomposition, and $\mathrm{Di}$ denotes detail components of decomposition. The subscript expresses the decomposition levels.

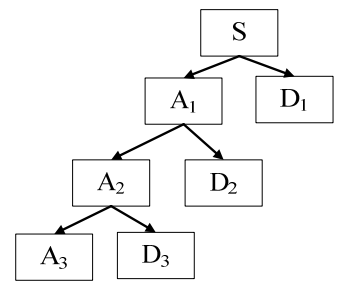

Figure 1. Tree diagram of 3 levels wavelet decomposition.

Reconstruction is the inverse process of wavelet decomposition. First of all, reconstruct the approximation and detail components on the lowest level to get approximation components of last level. Repeat this process till recovering the original signal. 
Between decomposition and reconstruction we may modify the wavelet coefficients before performing the reconstruction step. We perform wavelet analysis because the coefficients thus obtained have many known uses, denoising and compression being foremost among them.

\section{B. Frequency Localization of Wavelet Transform}

Through the definition of wavelet basic function, the graph center of the function is similar with a periodic function and attenuates quickly outside that, which can be considered as a window function with a certain frequency character as in [9]. If a signal's frequency is close to the wavelet basic function, the scale of the signal in the window will be amplified, attenuated on the contrary. With the shifts and expansions of wavelet basic function, we can recognize the signal's frequency characters.

Unlike the windowed Fourier transform, high frequency wavelets are very narrow, while low frequency wavelets are much broader. As a result, the wavelet transform is better able than the windowed Fourier transform to "zoom in" on very short-lived high frequency phenomena, such as transients in signals.

\section{Application in Random Error Separation}

The existing methods of random errors separation which using wavelet transforms mainly extract trends of the measurement data and get the random errors by subtracting the trend items, which is difficult to apply because it requires the precise modeling of the true values and fixed errors. The paper implements the separation from another perspective, which directly uses the details of wavelet transform approximate the random errors of measurement data, determines the wavelet decomposition levels based on the frequency bands character of the measurement data and separates the random errors directly which avoiding additional errors from the modeling process.

The new method of this paper need not know the error models that the random errors or the fixed errors satisfy with. The only thing we should pay attention to is setting up the relationship between the frequency bands character of the measurement data and the decomposition levels of the wavelet transform, and finding out the optimal decomposition level when the random errors has been separated entirely.

\section{METHOD OF RANDOM ERRORS SEPARATION}

\section{A. Frequency Bands Character of Measurement Data}

Uses the following mathematical model to express the measurement data as in [10]:

$$
y=f(t)+e
$$

Where $e$ is the random errors, $y$ is the measurement data, $f(t)$ is the other components except the random errors.

The actual signal usually has distinct spectrum distributions in different frequency band, so does the measurement data mentioned in [11]. In the model of Eq.1, frequency of the random errors $e$ is much higher than $f(t)$. There usually exists a band interval between $e$ and $f(t)$. Therefore, if set $G(L)=\frac{1}{n}\left(\sum_{k=1}^{n}\left(y_{k}-D_{k L}\right)^{2}\right)^{\frac{1}{2}}$, where $L$ is the wavelet decomposition levels, $D_{k L}$ is the $k t h$ details of level $L$ after wavelet decomposition, which contains the higher frequency signals of the decomposed data. The value of $G(L)$ expresses the differences between the measurement data and details of wavelet transform under the influence of decomposition levels $L$. With the increasing of the decomposition levels, due to the frequency bands character of the measurement data, the value of $G(L)$ will vary as follows:

$$
\begin{array}{ll}
G(L)>G(L+1), \quad L=1,2, \cdots, L_{1} \\
G(L) \approx G(L+1), \quad L=L_{1}+1, L_{1}+2, \cdots L_{2} \\
G(L)>G(L+1), \quad L=L_{2}+1, L_{2}+2, \cdots
\end{array}
$$

Eq.2, Eq.3 and Eq.4 show the approach process of detail components of wavelet transform approximating the random errors with the increasing of the decomposition levels. Approximate the random errors from level 1 to level $L_{2}$ gradually, and there are no other components except the random errors before the level $L_{2}$. Therefore, we can get the optimal decomposition level by calculating the value of $G(L)$ during the wavelet transform process.

\section{B. Random Errors Separation Processing}

Obviously it is the most important thing to determine the optimal decomposition levels. The paper proposes a new method which precisely determines the optimal decomposition level by analysis variety rules of the value of $G(L)$ based on the frequency bands character.

There are 4 steps to determine the optimal decomposition levels of random errors separation method by using bands character:

(1) Choose a wavelet basic function and decompose the measurement data to level 1 by wavelet transform;

(2) Calculate the value of $G(L)$ by using the detail components from wavelet transform, and judge whether $L$ is the optimal decomposition level or not;

(3) Repeat process (2) until getting the optimal decomposition level $L_{2}$;

(4) Reconstruct the detail components from level 1 to level $L_{2}$ to get the random errors of the measurement data, and then calculate mean square error of separated random errors.

Fig. 2 shows the flow chart of random errors separation process. 


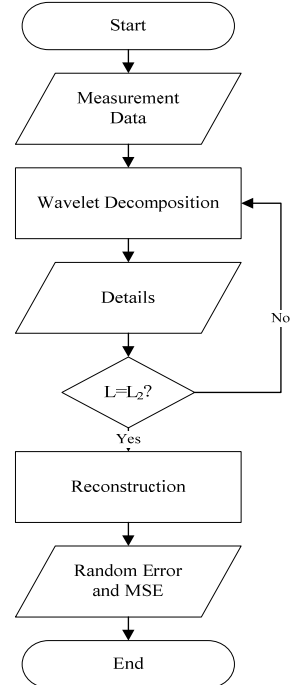

Figure 2. Flow chart of random errors separation process.

\section{EXPERIMENTAL RESULTS}

Assume measurement data $\{y(t): 0 \leq t \leq 1200 \times 0.05\}$ is from the following model

$$
\begin{aligned}
& y(t)=P(t)+\varepsilon(t) \\
& P(t)=\left\{\begin{array}{l}
\mathrm{P}_{0}(\mathrm{t}) \\
\mathrm{P}_{0}(\mathrm{t})-0.04(\mathrm{t}-20)^{2} \\
\mathrm{P}_{0}(\mathrm{t})-0.04(\mathrm{t}-20)^{2}+0.05(\mathrm{t}-40)^{2}
\end{array}\right.
\end{aligned}
$$

Where $\mathrm{P}_{0}(\mathrm{t})=1000+900 t+0.4 t^{2}+0.001 t^{3}, \varepsilon(t)$ is $20 \mathrm{~dB}$ white noise signal.

Choose Sym5 as the wavelet basic function, get trend of $G(L)$ by using the random errors separation methods, as shown in Fig. 3. From the Fig. 3(b) we can get the optimal decomposition level is 8 .

Reconstructing the detail components gets the separated random errors. Fig. 4 shows the result compare with the simulation error in time domain. The first figure plots the simulation random errors, the second figure plots the separated random errors, and the last one plots the difference between the two errors, which can observe that it is very close between the two random errors. Calculation results indicate the mean square error by using the paper method of random errors separation is $3.1754 \times 10^{3}$, the mean square error of simulation random errors is $3.1771 \times 10^{3}$. Fig. 5 shows the result comparison in frequency domain. Fig. 4 and Fig. 5 show that the method of this paper can separate the random errors from the measurement data in good accordance through the simulation test.

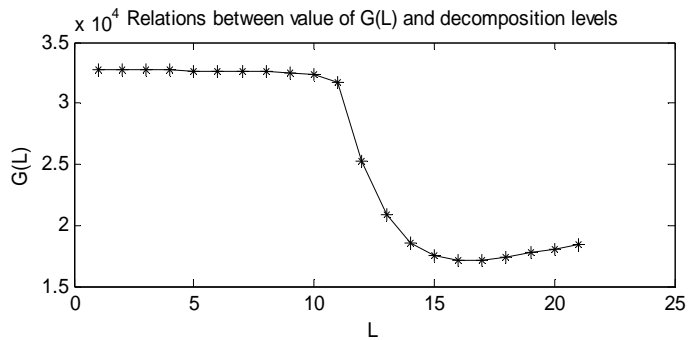

(a)

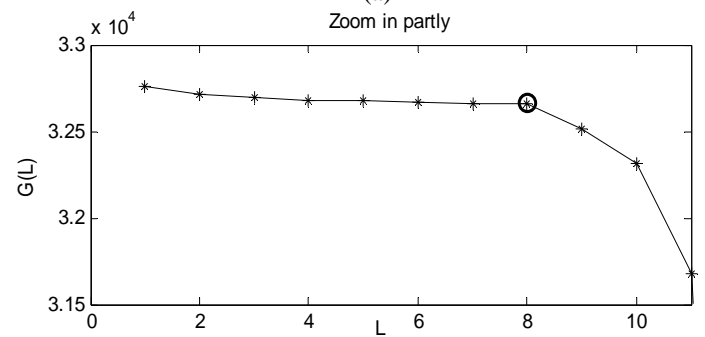

(b)

Figure 3. Relations between value of $G(L)$ and decomposition levels: (a) plots the panoramic graph, (b) plots the part one, which can get the changing trend more intuitionisticly.
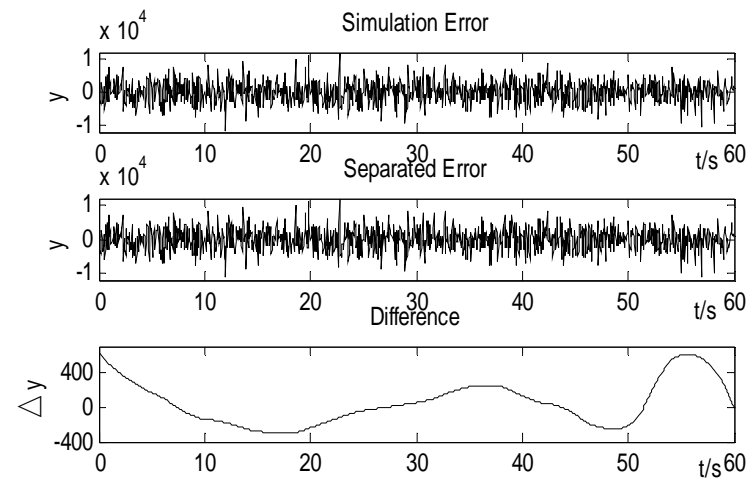

Figure 4. Comparison in time domain.
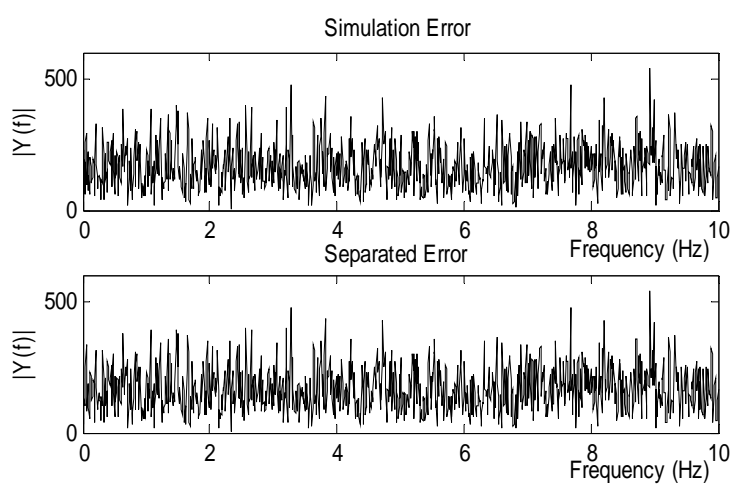

Figure 5. Comparison in frequency domain. 


\section{CONCLUSION}

Random errors separation is a basic job of the measurement data processing. In this paper, we have presented a new way of random errors separation different from the existing methods, which based on the frequency band intervals between the random errors and the true value together with the fixed errors. We can use detail components of wavelet transform to appropriate the random errors and determine the optimal wavelet decomposition levels by using the frequency bands character of the measurement data; finally reconstruct the detail components to implement random errors separation from the measurement data. The simulation results show that the wavelet transform in combination with measurement data bands character can separate the random errors separation simply and effectively.

\section{REFERENCES}

[1] Zhou Lifeng, Cao Shuyan, Jiang Dazhi, “A study on error analysis of radar measurement data in proving ranges,” Radar \& Ecm, 2008(2), pp. 4-7.

[2] Wang Xinlong, Du Yu, Ding Yangbin, "Investigation of random error model for fiber optic gyroscope,” Journal of Beijing University of Aeronautics and Astronautics, vol. 32, pp. 769-772, July 2006.

[3] Wang Hao, Huang Changqiang, Wang Yong, Zhao Hui, "Random error modeling of inertial sensors based on autoregressive process,"
Journal of Chinese Inertial Technology, vol. 16, pp. 224-227 February 2008.

[4] Jiang Qingxian, Wu Fumei, "Robust Estimation for FOG Data Based on Wavelet Threshold De-Noising," Chinese Journal of Sensors and Actuators, vol. 22, pp. 1596-1601, November 2009.

[5] Song Ningfang, Chen Jing, Jin Jing, "Wavelet variance analysis of random error properties for fiber optic gyroscope," Infrared and Laser Engineering, vol. 39, pp. 924-928, May 2010.

[6] Liu Lisheng, Post-Flight Data Processing of Trajectory Measurment, 1st ed., Beijing: National Defence Industry Press, 2000, pp. 298-300.

[7] Guo Lihua, Zhu Yuanchang, and Yin Wenlong, "Application of Wavelet Transform in Random Error Separation of Telemetered Data,” Journal of Projectiles, Rockets, Missiles and Guidance, vol. 31, pp. 249-252, August 2011.

[8] Xia Qing, Zhang Shifeng, and Cai Hong, "Analysis of Random Errors of Radar Measurement Data Based on Wavelet Transformation," Journal of Spacecraft TT\&C Technology, vol. 25, pp. 77-80, October 2006.

[9] Li Zhen, Zhu Feng, and Chen Jiajun, “Analysis and Processing of Wind Shock Deformation Monitoring Data for Bridge Based on Wavelet Transform,” Bulletin of Surveying and Mapping, vol. 18, pp. 18-20, November 2011.

[10] Tu Xianqin, Yi Dongyun, Yu Ningli, and Li Xiao-yong, "The Statistics of Measurement Noise of the Instrmentation Ship,” Journal of Spacecraft TT\&C Technology, vol. 25, pp. 72-77, June 2006.

[11] Yi Dongyun, Zhu Jubo, and Wang Zhengming, "Spline Method of Separating Signal with Three Frequency Bands," ACTA ELECTRONICA SINICA, vol. 27, pp. 54-57, January 1999. 